American Journal of Biochemistry and Biotechnology 4 (2): 167-176, 2008

ISSN 1553-3468

(C) 2008 Science Publications

\title{
Bridging from Cells to Cognition in Autism Pathophysiology: Biological Pathways to Defective Brain Function and Plasticity
}

\author{
${ }^{1}$ Matthew P. Anderson, ${ }^{2}$ Brian S. Hooker and ${ }^{3}$ Martha R. Herbert \\ ${ }^{1}$ Departments of Neurology and Pathology, Harvard Medical School/Beth Israel Deaconess Medical \\ Center, Harvard Institutes of Medicine, Room 846, 77 Avenue Louis Pasteur, Boston, MA 02115 USA \\ ${ }^{2}$ High Throughput Biology Team, Fundamental Science Directorate, Pacific Northwest National \\ Laboratory, 902 Battelle Blvd., Richland, WA 99354 USA \\ ${ }^{3}$ Pediatric Neurology/Center for Morphometric Analysis, Massachusetts General Hospital/Harvard \\ Medical School, 149 13th St., Room 6012, Charlestown, MA 02129 USA \\ and \\ Center for Child and Adolescent Development, Cambridge Health Alliance/Harvard Medical School, \\ 101 Station Landing, Room 2105, Medford, MA 02155 USA
}

\begin{abstract}
We review evidence to support a model where the disease process underlying autism may begin when an in utero or early postnatal environmental, infectious, seizure, or autoimmune insult triggers an immune response that increases reactive oxygen species (ROS) production in the brain that leads to DNA damage (nuclear and mitochondrial) and metabolic enzyme blockade and that these inflammatory and oxidative stressors persist beyond early development (with potential further exacerbations), producing ongoing functional consequences. In organs with a high metabolic demand such as the central nervous system, the continued use of mitochondria with damaged DNA and impaired metabolic enzyme function may generate additional ROS which will cause persistent activation of the innate immune system leading to more ROS production. Such a mechanism would self-sustain and possibly progressively worsen. The mitochondrial dysfunction and altered redox signal transduction pathways found in autism would conspire to activate both astroglia and microglia. These activated cells can then initiate a broad-spectrum proinflammatory gene response. Beyond the direct effects of ROS on neuronal function, receptors on neurons that bind the inflammatory mediators may serve to inhibit neuronal signaling to protect them from excitotoxic damage during various pathologic insults (e.g., infection). In autism, over-zealous neuroinflammatory responses could not only influence neural developmental processes, but may more significantly impair neural signaling involved in cognition in an ongoing fashion. This model makes specific predictions in patients and experimental animal models and suggests a number of targets sites of intervention. Our model of potentially reversible pathophysiological mechanisms in autism motivates our hope that effective therapies may soon appear on the horizon.
\end{abstract}

Key words: Mitochondria, ROS, BDNF, dendritic beading, synaptic plasticity, inflammation

\section{INTRODUCTION}

An emerging focus in autism research on metabolic and tissue properties of living brains marks a move beyond methodologies and models that to date have predominantly been applied to determining the brain basis of autism. Approaches to the brain in autism have been conditioned by inferences drawn from a number of considerations: 1) the presence of a set of behaviors that are strongly associated in a syndrome, 2) high monozygotic concordance and recurrence rates and 3) physical evidence in neuropathological and brain imaging studies of neuroanatomical abnormalities, some of which are consistent with prenatal onset. The inferences drawn from these considerations have until recently largely focused on the model of a geneticallybased disorder of prenatal pathogenesis where altered development has led to structural abnormalities that

Corresponding Author: Matthew P. Anderson, Departments of Neurology and Pathology,

Harvard Medical School/Beth Israel Deaconess Medical Center, Harvard Institutes of Medicine, Room 846, 77 Avenue Louis Pasteur, Boston, MA 02115 USA, Tel: 6176670853 
underlie functional aberrations. These assumptions have driven a research agenda strongly biased towards seeking mutations in developmental genes and identifying structural abnormalities in brain regions that might plausibly be associated with identified neurocognitive impairments, as well as for mechanisms that might lead to neuroanatomical alterations occurring early in development.

However this research program has not delivered findings that clearly support these inferences. No genes of strong effect have been identified other than possibly a small number of syndromic autism cases (e.g. Fragile $\mathrm{X}$ ), yet even in those cases the specific modulatory mechanisms underlying the lack of full penetrance have not been elucidated ${ }^{[1]}$. Additionally, brain changes of various types have been documented to exist during postnatal period, including increasing brain volume $\mathrm{e}^{[2,3]}$, persistent immune activation ${ }^{[4]}$ and diminution of cell size $^{[4]}$, with the latter two continuing substantially into the lifespan. With genes of strongest effect "causing" autism in less than half of individuals carrying such genes and with the recognition of postnatal pathophysiologies, the progress of neurobiology is undermining the arguments for construing autism as a purely prenatally determined brain disorder. In fact, discourse on autism is increasingly including not only the term neurodevelopmental but also the term neurodegenerative in characterizing the disorder.

Yet even such postnatal changes as have been documented in autism are not of the character or severity that would be expected in a severe and relentless neurodegenerative process. Although the steeply rapid pace of very early brain enlargement does not continue past the first year or two of postnatal life ${ }^{[2]}$ and although there may conceivably be mild volume loss into the middle of the lifespan ${ }^{[5]}$, major volume loss, massive cell death or brain atrophy is not seen. Furthermore, there is currently no evidence at all that these volume changes (increase or loss) are due to the process that is the basis for autism. Neither have we seen to date an appreciable shortening of the lifespan due to the disease process itself ${ }^{[6]}$. Thus we cannot call upon mechanisms of frank tissue destruction to account for autism's functional deficits.

The notion of abnormal brain connectivity that is now being fleshed out in an expanding research domain poses yet another challenge to the assumptions that have up until recently dominated autism brain research. This notion, discussed as early as the $1960 \mathrm{~s}^{[7]}$ and $1980 \mathrm{~s}^{[8]}$, has reemerged in the setting of technologies facilitating its measurement as the idea that covariance or co-activation between brain regions in autism is altered or reduced in a widely distributed fashion ${ }^{[9,10]}$.
Placed alongside of documented brain enlargement and in particular of white matter enlargement, it is tempting to attribute altered connectivity to altered distribution of axons, with an increase in short-range but a decrease in long-range connectivity explaining altered processing patterns being documented by functional neuroimaging and electrophysiology. ${ }^{[11,12]}$ However, given that the anatomical enlargement does not persist into adulthood whereas altered functional connectivity has been measured in adults, it bears reflection that this altered functional connectivity may not be based primarily in macroanatomical alterations. Moreover, the small amount of tissue-oriented imaging studies published to date that attempt to characterize the physical changes underlying autism have brought into question whether the areas documented as enlarged have more axons, thicker myelination (oligodendroglial hypertrophy), astroglial hypertrophy, microglial hypertophy, or simply increased or altered fluid content ${ }^{[13]}$. Furthermore, specific mechanisms by which such structural changes might directly relate to altered function have not been established.

The possibility might still remain that microanatomical architectural abnormalities may be the driving force underlying the autistic functional deficits. Abnormalities in receptors, interneurons, Purkinje cells, dendritic arborization, minicolumns and other microscopic abnormalities are abundant in autism literature ${ }^{[14,15]}$. Even here, however, debate has arisen on a number of points. First, interpretations of such abnormalities as being prenatal have been challenged by arguments proffering mechanisms by which at least some of these changes could occur due to postnatal phenomena. For instance, minicolumnar abnormalities could arise early in gestation but they might also be a consequence of postnatal nitric oxide abnormalities ${ }^{[16]}$, while Purkinje cell dropout could result from postnatal excitotoxicity ${ }^{[17]}$. Yet, there are always intrinsic problems posed by working with human postmortem material and repeatedly throughout history such patterns have been associated with a variety of other neurologic conditions including for example infantile spasms. Second, it has not been adequately assessed whether the microanatomical abnormalities documented to date are static architectural endpoints or whether they might be potentially dynamic conditions that are being modulated by chronic features of autism, such as immunity or oxidative stress, but that might rebound were the chronic aggravating stressors to abate. Regarding Purkinje cells, the recent findings that these cells stain with calbindin but not with Nissl has been interpreted as being a consequence of agonal or postmortem changes ${ }^{[18]}$, but it has not been excluded 
that the problem could have been with the status of these cells before death and that the poor uptake of Nissl stain might instead reflect an ongoing stress response (e.g., chromatolysis, a depletion of somatic rough endoplasmic reticulum) related to chronically diseased and weakened cells during life.

Substantial clinical observation supports the existence of considerable intra-individual variability in at least some children with autism. This has been poorly studied, presumably because the research agenda has been focused on autism as a condition that is stable across the life course. Research attention is now starting to be paid to those children whose autism becomes less severe over time as well as to those who lose their autism diagnosis ${ }^{[19,20]}$. Also being investigated are intriguing transient changes in severity, such as fairly common reports that some children in the setting of high fever show improvements in eye contact and communication skills that then are lost when the fever subsides $^{[21-24]}$. These clinical observations and the latter ones in particular, suggest that at least some components of autism may in at least some cases be reversible and related to a neuromodulator state. While these neuromodulators may act upon an altered macroor micro-anatomical substrate, it nevertheless remains the case that such relatively stable substrate alterations alone could not explain this kind of changeability.

Intra-individual clinical variability adds a new dimension to the problem of studying geneenvironment interactions. Emphasis to date has been upon vulnerable neurodevelopmental mechanisms, with xenobiotics, infectious agents and autoimmune processes and their immunologic consequences having an impact on brain development. While our model includes intra-uterine maternal insults (Fig. 1), whether these insults persist into adulthood, are structural or molecular and are irreversible or reversible remains open for debate. Many of the signaling molecules and other mechanisms by which environmental agents may affect neurodevelopment could also modulate function in an already developed brain. Although some postnatal development continues into early childhood, autistic regression of the infant argues in favor of a functional model with potential reversibility.

While such potentially reversible mechanisms have not been the focus of autism research, enough is known about these cellular and molecular processes to at least sketch out a plausible research program oriented towards identifying modulators of neural function and plasticity that may be chronic and yet under certain circumstances also variable in their severity and conceivably also even treatable.

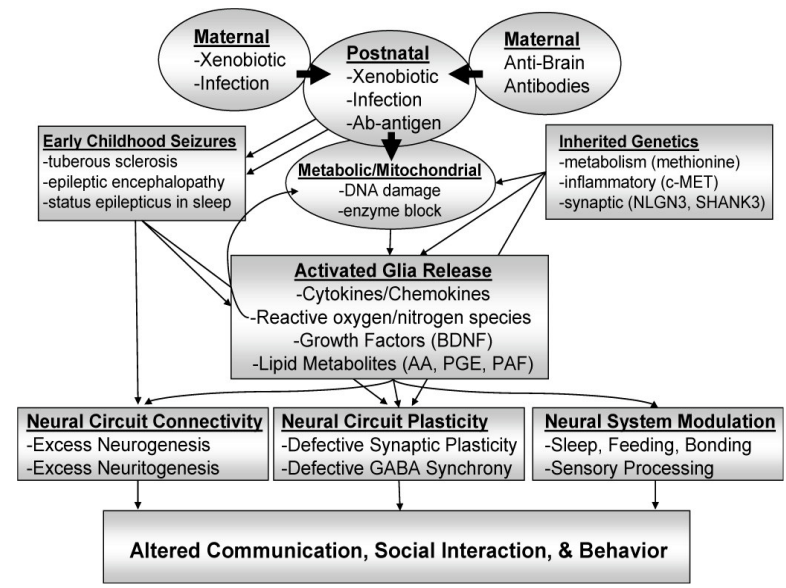

Fig. 1: An overview of the various classes of cellular, environmental, and genetic factors potentially leading to the behavioral defects in language, social behavior, and sensory processing

Mechanisms: From the vantage point described above, evidence from the metabolic and molecular levels can be reviewed to highlight dimensions of chronic and functional as well as developmental and structural features pertinent to behavioral, processing and biological phenotypic characteristics that have been measured as different from typical function in autism.

Within the set of interlocking mitochondrial, inflammatory and molecular mechanisms that have been associated with autism, there appear to be a number of phenomena that could readily associate with neuronal dysfunction rather than cell death. Particularly pertinent is the interrelationship of mitochondrial dysfunction, neuroinflammation and neuronal dysfunction diagramed in our model (Fig. 1). Mitochondrial dysfunction has been reported in a significant fraction of autistic patients ${ }^{[25-27]}$. Activated microglia, also documented in autism, ${ }^{[28]}$ produce an excess of reactive oxygen species and reactive nitrogen species, including superoxide, nitric oxide and peroxynitrite $^{[29-32]}$, which subsequently can lead to mitochondrial DNA damage or protein modifications that cause mitochondrial dysfunction. Impaired methionine metabolism has also been described in autism and a hyperaccumulation of homocysteine that can be associated with such impairments has been shown in rat cerebellar granule cells to lead to mitochondrial swelling and cytochrome $\mathrm{c}$ release that occurs in a calcium concentration-independent manner ${ }^{[33]}$. An earlier report implicated DNA damage caused by high homocysteine levels in activating mitochondrial disruption in rat hippocampal cell 
cultures following a sequence of DNA breakage, polyADP-ribose polymerase (PARP) activation, caspase activation, p53 activation and loss of mitochondrial membrane potential ${ }^{[34]}$. DNA breakage associated with hyperhomocysteinemia may be due to subsequent DNA undermethylation and accordingly uracil misincorporation $^{[35]}$. It is notable that these processes were directly reversed by inhibiting PARP.

In utero infections have been associated with a postnatal risk for neuropsychiatric symptomatology independent of the specific infectious agent ${ }^{[36]}$. Beyond this, evidence in the literature suggests that in the setting of maternal inflammation alone, the child may sustain long-standing mitochondrial DNA damage and depletion. HIV negative children born to untreated HIV+ mothers displayed major mitochondrial DNA damage (40\% depletion) that persisted throughout the two postnatal years during which observations were made ${ }^{[37]}$. While the incidence of autism in children born to HIV+ mothers has not been examined, the data do suggest that any inflammatory condition in the mother might conceivably promote severe mitochondrial (and possibly nuclear) DNA damage to her child. The mechanism of this effect is suspected to occur as follows. Maternally infected cells would promote an increased production of inflammatory cytokines that cross the placenta and then increase interleukin $1 \beta$, interleukin 6 , TNF- $\alpha$ and interferon- $\beta$ among others, by fetal cells ${ }^{[38]}$. Mitochondrial DNA fragmentation may then be induced by free radical production associated with the increase in these cytokines, especially interferon- $\beta$. The impact of this damage on nuclear and mitochondrial DNA damage in the neuron could be even more severe due to the high neuronal energy consumption rate and the lack of cell turnover. Due to the positive feed-back loops formed in such a mechanism (Fig. 1), the disease state could self-sustain and persist well into later life. Such a model could have relevance to a variety of disease processes.

Genetic and molecular changes that have been described in autism and interpreted developmentally may also have chronic and ongoing features some of which moreover may be modifiable. A polymorphism in the upstream region of the hepatocyte growth factor receptor c-Met that has been identified in a study involving 1231 autistic children plus appropriate controls $^{[39]}$ has the dual consequences of a deficiency in c-Met protein level corresponding to reduced transcription rate and a downregulation of hepatocyte growth factor (HGF) production as c-MET/HGF signaling is directly related to $\mathrm{HGF}$ expression via a positive feedback mechanism ${ }^{[40,41]}$. It is thus noteworthy that HGF levels have also been shown to be lower in high functioning autistic adult males ${ }^{[42]}$. This raises the question of whether biological activity of HGF contributes to autism symptomatology in a chronic fashion. Interestingly, studies suggest c-MET might mediate reversal of the microgliosis and astrogliosis in post-ischemic repair as evidenced by anti-apoptosis and the prevention of glial scar formation ${ }^{[43-45]}$, while HGF deficiencies were connected to an increased oxidative stress response $^{[46]}$. This latter connection is mediated by a mechanism in which specificity protein 1 (Sp1), a transcription factor that initiates c-MET expression, is inhibited in the presence of $\operatorname{ROS}^{[46,47]}$. Sp1 inhibition is caused by the expression of Early growth factor 1 (Egr1) which binds the transcription factor competitively with the c-MET upstream initiation region. Egr-1 expression is stimulated by ROS as demonstrated via the addition of $\mathrm{H}_{2} \mathrm{O}_{2}{ }^{[48]}$ as well as methamphetamines which cause ROS and free radical accumulation in the brain $^{[49]}$. Furthermore, HGF and c-MET both appear to be pertinent beyond the brain, since HGF reduces inflammation in other tissues ${ }^{[50,51]}$ and can potently suppress dendritic cell functions ${ }^{[52]}$. Thus deficiencies of both c-MET and HGF lead to conditions that would promote inflammation or oxidative stress which would indeed increase the frequency of DNA breakage. Thus, less c-MET could mean less control of inflammation (i.e., more chronic inflammation), more ROS, more caspase-mediated damage and more uracil misincorporation. It is hence worth dwelling upon the ways that a deficiency in either HGF or c-MET could initiate and also potentially promote a sustained immune response across the life course to sustain processes that are consistent with chronic features of autism pathophysiology and could therefore potentially be an important contributor to the autism phenotype.

Function: How might these persistent metabolic and molecular dysfunctions and deficiencies lead to neural systems dysfunction? The persistent activation of innate immunity in the CNS as observed in autism could cause ongoing impairments of neural circuit function through numerous mechanisms independent of its possible effects on cortical column number and pattern. In fact, considering the large number of cytokines, chemokines and lipid metabolites found to be elevated in the CSF and tissue from the brain of individuals with autism ${ }^{[4]}$, one would be surprised if these did not cause any effect on circuit function. Suppression of neural circuit function during an innate immune response may have developed as a defense against excitotoxic cell death and other forms of permanent cell (e.g., mitochondrial or nuclear DNA damage) and circuit damage. These neural tissue 
responses would also be designed to be readily reversible, whether through simple down-regulation of inhibitory transcripts and proteins or through reformation of temporarily disconnected synaptic contacts.

At the neural circuit level, there are numerous molecular mechanisms whereby innate immune inflammation might impair function of circuits that need not rely on disturbances of neuronal progenitor migration or axonal arbor development (growth, targeting or pruning) and moreover such neural circuit level functional mechanisms deserve attention because of potentially reversible features. We present this model of reversible functional defects as an alternative view point to the dominant paradigm in autism research. As described in earlier paragraphs, consideration of such alternative models is motivated by the evidence of reversibility, regression and ongoing CNS inflammation in autism. However, we must point out that this is simply an alternative view point and developmental models could also be built to explain the behavioral features observed in autism research.

Innate immune inflammation of the brain causes severe disturbances of cognition. Dementia, a deficit in learning and memory, develops in the context of innate immune inflammation of the CNS associated in human immunodeficiency virus (HIV) encephalopathy. This retroviral infection of microglia triggers a persistent innate immune inflammation within the CNS characterized by a mild-to-moderate microglial and astroglial hypertrophy without causing any evidence of cell death or damage. Of note and by analogy to HIV encephalopathy, in autism, cell death is not observed and cell damage is subtle and the brains macroscopically and microscopically for the most part look clinically normal with the exception of a mild-tomoderate glial response ${ }^{[15]}$. In HIV, careful examination of golgi-stained cortical neurons did eventually reveal a pattern of dendrite beading not seen in control brains ${ }^{[53]}$. Dendritic beading has been observed in a variety of disorders including epilepsy and Alzhiemer's disease, is triggered by NMDA receptor agonists and depends on sodium influx ${ }^{[54-56]}$. In vitro studies of brain slices have been used to try to investigate the consequences of this morphologic change of dendrites. Treatment of brain slices with NMDA receptor agonists trigger the same pattern of dendritic beading and the response is potentiated by the inflammatory mediator lipid metabolite platelet-activating factor (PAF) that is found at high concentrations in the CSF of individuals with AIDS encephalopathy ${ }^{[57]}$. The precise signal transduction pathway and intracellular mediators remain largely undefined. Functionally, the formation of dendritic beads is associated with reduced synaptic long-term potentiation (LTP) in some models and chronic synaptic depression in others ${ }^{[57,58]}$. Synaptic LTP has been shown to be critical to learning and memory ${ }^{[59,60]}$. Consequently, one might speculate that inflammatory mediator-induced dendritic beading and impaired LTP might prevent the synaptic encoding of language and social response patterns as a potential basis for the communication deficits seen in autism spectrum disorder. Dendritic beading has not yet been described in the autism neuropathology literature, but this same change was not initially recognized in HIV encephalopathy ${ }^{[61]}$.

Innate immune inflammation could also impair synaptic plasticity by impairing neuronal synchrony or promoting aberrant forms of neuronal synchrony. Seminal experiments in the late 1990s demonstrated that presynaptic and postsynaptic neurons must fire within $<100 \mathrm{~ms}$ of each other to undergo synaptic plasticity. Furthermore, reversing the order of firing inverted the plasticity from potentiating to depotentiating ${ }^{[62-65]}$. Based on this principle, impaired synchronized firing of glutamatergic neurons could in principle produce aberrant synaptic plasticity. GABAergic interneurons are interconnected by electrical gap junctions that enable them to form an electrical syncitium which synchronizes their firing and the firing of their targets the glutamatergic pyramidal neurons ${ }^{[6]}$. In hippocampus, minimal stimulation evokes inhibitory synaptic currents which synchronize the firing of populations of CA1 pyramidal neurons ${ }^{[67]}$. Chronic inflammation in the brain of an individual with autism might impair the ability of GABAergic networks to synchronize the firing of pyramidal neurons and to facilitate spike-timing dependent synaptic plasticity. A potential basis for impaired GABAergic synaptic transmission is suggested by recent studies on the impact of microglia on GABA synapses in pain research. In studies of neurogenic pain, chronic inflammation due to microglial activation is generated by traumatic spinal cord injury. Evidence suggests activated microglia within the spinal cord release BDNF which collapses the anion gradient ${ }^{[68]}$. BDNF via TrkB receptors down regulates the $\mathrm{K}+-\mathrm{Cl}^{-}$cotransporter $\mathrm{KCC} 2$ which impairs removal of intracellular $\left(\mathrm{Cl}^{-}\right)^{[69]}$. Loss of this chloride gradient would eliminate the hyperpolarizing currents evoked by GABAergic synapses. The spike synchronization described by Cobb et al. ${ }^{[67]}$ in the hippocampus depends on the ability of the GABAergic synapse to transiently hyperpolarize the membrane of a large number of CA1 pyramidal neurons causing a rebound depolarization through active conductances (e.g., T-type $\mathrm{Ca}^{2+}$ 
channels ${ }^{[70]}$ ) that trigger synchronous firing of action potentials. Synchronizing the firing of neurons with collateral connections (e.g., layer II/III cortical or CA3 hippocampal pyramidal neurons) will promote spiketiming-dependent synaptic plasticity. The failure of such mechanisms could be used to explain the "underconnectivity" that has been observed at the level of macroscopic circuitry level in autism ${ }^{[9,10]}$.

Applying the same concepts of spike-timing synaptic plasticity, one should note that by simply inverting the order of synchronous firing, one could also erase critical information previously encoded via synaptic potentiation. Aberrant patterns of synchronous circuit activity that occur in continuous spike-and-wave epilepsy of sleep result in a pattern of language and sometimes more global autistic regression ${ }^{[1,72]}$. We speculate that the issues go beyond the potential problems for skill acquisition caused by the failure to synchronize firing, since aberrant synchronization of firing may in fact depotentiate synapses in these children to erase the synaptic plasticity events that encode learned behaviors associated with major developmental milestones.

These represent just two examples of mechanisms whereby chronic innate immune inflammation could impair synaptic plasticity. Some of the cytokines, chemokines, or other inflammatory mediators could also regulate the central neuromodulatory systems (dopaminergic, orexinergic, histaminergic, serotonergic, noradrenergic, cholinergic) in the brain to not only influence plasticity but also produce major disturbances in sleep-wake cycle, feeding, or social behavior ${ }^{[73-75]}$ that are notable parts of the autism phenotype.

As described above, there is ample evidence for defects in mitochondrial function in autism. In some cases, there are autism-associated mutations in genes implicated in mitochondrial metabolism and in other cases environmental toxins which interfere with key enzymes involved in mitochondrial function. Are there neural functions that could bridge directly from neuronal mitochondrial dysfunction to behavioral deficits, seizures, or sensory pathologies associated with autism independent of the inflammatory mediator effects? One potential clue is the observation that collapsing the mitochondria membrane potential impairs the clearance of intracellular calcium in a large presynaptic terminus, the Calyx of Held ${ }^{[76]}$. Although it is unclear whether mitochondrial defects cause similar effects in other major glutamatergic synapses, synaptic transmitter release was decreased by $50 \%$ when mitochondrial function was impaired. We predict that any neuron that must sustain high firing rate such as a fast-spiking GABAergic interneuron or a thalamocortical sensory relay neuron $(150-200 \mathrm{~Hz}$, M.R. Kasten and M.P. Anderson, in preparation), transmission may be particularly prone to failure in energy deficiency states. Failures of GABAergic synaptic transmission could unleash seizures and potentially cause regression in individuals as observed in epileptic encephalopathy. Failure of thalamocortical neuron sensory transmission might impair sensory map formation in the cortex or impair the synaptic plasticity of sensory-motor circuits needed for language acquisition.

Development of a mammalian model of the effects of innate immune inflammation on neural circuit physiology and plasticity will be necessary to define which signaling molecules are the major players and which neural circuit mechanisms are impaired. Experimental models can be developed to systematically address multiple dimensions of an integrative model (Fig. 1) ${ }^{[73]}$. By performing these studies in the mouse, one can not only create autismassociated gene mutations to model genetic components of the disease, but also use the power of conditional genetics (Cre/loxP and other technologies) to intervene on specific genes and neural circuitries (e.g., cortex vs. thalamus ${ }^{[70]}$ ) to define precisely where inflammation acts to impair function and behavior. The detailed mechanistic insights into autism pathophysiology that can be obtained through these approaches will almost certainly inspire novel ideas in the treatment for this life-long neurological condition that affects so many families today.

\section{CONCLUSION}

The above considerations suggest a number of directions for model-building, hypothesis formulation and research. First, the shift from a prenatal and developmental perspective to a view that also includes ongoing and chronic pathophysiology raises the question of the functional pertinence of mechanisms that persist past the periods of most active brain development. Second, the shift from a static to a dynamic formulation of autism allows a shift in perspective on the source of intra-individual variability. Rather than considering it to be an idiosyncratic rippling over an unchanging set of core autistic features, the phenomenon is considered potentially central to our understanding of autism pathophysiology providing a new playing field for exploring its neurobiological foundations. And third, conceptions of potential treatment targets can be expanded to include modulation of mechanisms that may be genuinely 
reversible and not just addressed in a compensatory fashion.

Pursuit of these novel horizons will require the coupling of a number of levels of investigation that hitherto have been little explored in autism research, particularly in combination. It will require a hypothesis-driven coupling of immune, metabolic/biochemical and neurophysiological investigations and it will also require in vivo pathophysiological investigations. As these methodologies will allow the exploration of potential avenues of plasticity in autism, implications for medical treatment should motivate the implementation of this research program.

\section{ACKNOWLEDGEMENT}

MPA was supported by funding from the National Alliance on Autism Research, the National Institute of Mental Health, the National Institute of Neurologic Disease and Stroke and the Burroughs Wellcome Fund. MRH was supported by NINDS NS048455, Cure Autism Now Foundation, Autism Speaks and the Bernard Fund for Autism Research. We thank Melissa W. Anderson for editing the manuscript and for administrative support.

\section{REFERENCES}

1. Belmonte, M.K. and T. Bourgeron, 2006. Fragile X syndrome and autism at the intersection of genetic and neural networks. Nat. Neurosci., 9: 1221-1225.

2. Redcay, E. and E. Courchesne, 2005. When is the brain enlarged in autism? A meta-analysis of all brain size reports. Biol. Psychiatry, 58: 1-9.

3. Herbert, M.R., 2005. Large brains in autism: the challenge of pervasive abnormality. Neuroscientist, 11: 417-440.

4. Bauman, M.L. and T.L. Kemper, 2005. Neuroanatomic observations of the brain in autism: a review and future directions. Int. J. Dev. Neurosci., 23: 183-187.

5. Aylward, E.H., N.J. Minshew, K. Field, B.F. Sparks and N. Singh, 2002. Effects of age on brain volume and head circumference in autism. Neurology, 59: 175-183.

6. Shavelle, R.M., D.J. Strauss and J. Pickett, 2001. Causes of death in autism. J. Autism Dev. Disord., 31: 569-576.

7. Rimland, B., Infantile autism; the syndrome and its implications for a neural theory of behavior. 1964, [New York],: Appleton-Century-Crofts. xi, pp: 282 .
8. Horwitz, B., J.M. Rumsey, C.L. Grady and S.I. Rapoport, 1988. The cerebral metabolic landscape in autism: Intercorrelations of regional glucose utilization. Arch. Neurol., 45: 749-755.

9. Just, M.A., V.L. Cherkassky, T.A. Keller and N.J. Minshew, 2004. Cortical activation and synchronization during sentence comprehension in high-functioning autism: evidence of underconnectivity. Brain, 127: 1811-1821.

10. Rippon, G., J. Brock, C. Brown and J. Boucher, 2007. Disordered connectivity in the autistic brain: challenges for the new psychophysiology. Int. J. Psychophysiol., 63: 164-172.

11. Courchesne, E. and K. Pierce, 2005. Why the frontal cortex in autism might be talking only to itself: local over-connectivity but long-distance disconnection. Curr. Opin. Neurobiol., 15: 225230.

12. Belmonte, M.K., G. Allen, A. Beckel-Mitchener, L.M. Boulanger, R.A. Carper and S.J. Webb, 2004. Autism and abnormal development of brain connectivity. J. Neurosci., 24: 9228-9231.

13. Hendry, J., T. DeVito, N. Gelman, M. Densmore, N. Rajakumar, W. Pavlosky, P.C. Williamson, P.M. Thompson, D.J. Drost and R. Nicolson, 2006. White matter abnormalities in autism detected through transverse relaxation time imaging. Neuroimage, 29: 1049-1057.

14. Palmen, S.J., H. van Engeland, P.R. Hof and C. Schmitz, 2004. Neuropathological findings in autism. Brain, 127: 2572-2583.

15. Herbert, M.R. and V.S. Caviness, Autism: A neurobiological disorder of early brain development, in Neuroanatomy and Imaging Studies, R. Tuchman and I. Rapin, Editors. 2006, Mac Keith Press. 115-140.

16. Gustafsson, L., 2004. Comment on Disruption in the inhibitory architecture of the cell minicolumn: implications for autism. Neuroscientist, 10: 189191.

17. Kern, J.K., 2003. Purkinje cell vulnerability and autism: a possible etiological connection. Brain Dev., 25: 377-382.

18. Kemper, T.L., M.L. Bauman and G.J. Blatt, 2004. Calcium-binding proteins in cerebellar Purkinje cells in the autistic cerebellum. in Society for Neurosciences.

19. Kelley, E., D. Fein and L. Naigles, 2007. Language functioning in optimal outcome children with a history of autism spectrum disorders. J. Autism Dev. Disord., In press. 
20. Fein, D., P. Dixon, J. Paul and H. Levin, 2005. Brief report: pervasive developmental disorder can evolve into ADHD: case illustrations. J Autism Dev. Disord., 35: 525-534.

21. Curran, L.K., et al., 2007. Behaviors associated with fever in children with autism spectrum disorders. Pediatrics, In Press.

22. Brown, G., 1999. The sometimes son - fever may temporarily cure autism. Humanist, 59: 46-47.

23. Cotterill, R.M., 1985. Fever in autistics. Nature, 313: 426.

24. Cotterill, R.M., 1986.The Brain: An Intriguing Piece of Condensed Matter. Physica Scripta, T13: 161-168.

25. Oliveira, G., L. Diogo, M. Grazina, P. Garcia, A. Ataide, C. Marques, T. Miguel, L. Borges, A.M. Vicente and C.R. Oliveira, 2005. Mitochondrial dysfunction in autism spectrum disorders: a population-based study. Dev. Med. Child Neurol., 47: 185-189.

26. Correia, C., A.M. Coutinho, L. Diogo, M. Grazina, C. Marques, T. Miguel, A. Ataide, J. Almeida, L. Borges, C. Oliveira, G. Oliveira and A.M. Vicente, 2006. Brief report: High frequency of biochemical markers for mitochondrial dysfunction in autism: no association with the mitochondrial aspartate/glutamate carrier SLC25A12 gene. J. Autism Dev. Disord., 36: 1137-1140.

27. Filipek, P.A., J. Juranek, M.T. Nguyen, C. Cummings and J.J. Gargus, 2004. Relative carnitine deficiency in autism. J. Autism Dev. Disord., 34: 615-623.

28. Vargas, D.L., C. Nascimbene, C. Krishnan, A.W. Zimmerman and C.A. Pardo, 2005. Neuroglial activation and neuroinflammation in the brain of patients with autism. Ann. Neuro., 57: 6781.

29. Colton, C.A. and D.L. Gilbert, 1987. Production of superoxide anions by a CNS macrophage, the microglia. FEBS Lett., 223: 284-288.

30. Moss, D.W. and T.E. Bates, 2001. Activation of murine microglial cell lines by lipopolysaccharide and interferon-gamma causes NO-mediated decreases in mitochondrial and cellular function. Eur. J. Neurosci., 13: 529-538.

31. Liu, B., H.M. Gao, J.Y. Wang, G.H. Jeohn, C.L. Cooper and J.S. Hong, 2002. Role of nitric oxide in inflammation-mediated neurodegeneration. Ann. N. Y. Acad. Sci., 962: 318-31.
32. Li, J., O. Baud, T. Vartanian, J.J. Volpe and P.A. Rosenberg, 2005. Peroxynitrite generated by inducible nitric oxide synthase and NADPH oxidase mediates microglial toxicity to oligodendrocytes. Proc. Natl. Acad. Sci. USA, 102: 9936-9941.

33. Zieminska, E., E. Matyja, H. Kozlowska, A. Stafiej and J.W. Lazarewicz, 2006. Excitotoxic neuronal injury in acute homocysteine neurotoxicity: role of calcium and mitochondrial alterations. Neurochem. Int., 48: 491-497.

34. Kruman, I.I., C. Culmsee, S.L. Chan, Y. Kruman, Z. Guo, L. Penix and M.P. Mattson, 2000. Homocysteine elicits a DNA damage response in neurons that promotes apoptosis and hypersensitivity to excitotoxicity. J. Neurosci., 20: 6920-6926.

35. Blount, B.C., M.M. Mack, C.M. Wehr, J.T. MacGregor, R.A. Hiatt, G. Wang, S.N. Wickramasinghe, R.B. Everson and B.N. Ames, 1997. Folate deficiency causes uracil misincorporation into human DNA and chromosome breakage: implications for cancer and neuronal damage. Proc. Natl. Acad. Sci. USA, 94: 3290-3295.

36. Patterson, P.H., 2002. Maternal infection: window on neuroimmune interactions in fetal brain development and mental illness. Curr. Opin. Neurobiol., 12: 115-118.

37. Poirier, M.C., R.L. Divi, L. Al-Harthi, O.A. Olivero, V. Nguyen, B. Walker, A.L. Landay, V.E. Walker, M. Charurat, W.A. Blattner and Women and Infants Transmission Study (WITS) Group, 2003. Long-term mitochondrial toxicity in HIV-uninfected infants born to HIV-infected mothers. J. Acquir. Immune Defic. Syndr., 33: 175183.

38. Ohyama, K., T. Sano and H. Toyoda, 2004. Predominant contribution of IFN-beta expression to apoptosis induction in human uterine cervical fibroblast cells by influenza-virus infection. Biol. Pharm. Bull., 27: 1750-1757.

39. Campbell, D.B., J.S. Sutcliffe, P.J. Ebert, R. Militerni, C. Bravaccio, S. Trillo, M. Elia, C. Schneider, R. Melmed, R. Sacco, A.M. Persico and P. Levitt, 2006. A genetic variant that disrupts MET transcription is associated with autism. Proc. Natl. Acad. Sci. USA, 103: 16834-16839.

40. Yo, Y., R. Morishita, K. Yamamoto, N. Tomita, I. Kida, S. Hayashi, A. Moriguchi, S. Kato, K. Matsumoto, T. Nakamura, J. Higaki and T. Ogihara, 1998. Actions of hepatocyte growth factor as a local modulator in the kidney: potential role in pathogenesis of renal disease. Kidney Int., 53: $50-58$. 
41. Elliott, B.E., W.L. Hung, A.H. Boag and A.B. Tuck, 2002. The role of hepatocyte growth factor (scatter factor) in epithelial-mesenchymal transition and breast cancer. Can. J. Physiol. Pharmacol., 80: 91-102.

42. Sugihara, G., K. Hashimoto, Y. Iwata, K. Nakamura, M. Tsujii, K.J. Tsuchiya, Y. Sekine, K. Suzuki, S. Suda, H. Matsuzaki, M. Kawai, Y. Minabe, A. Yagi, N. Takei, T. Sugiyama and N. Mori, 2007. Decreased serum levels of hepatocyte growth factor in male adults with highfunctioning autism. Prog Neuropsychopharmacol Biol. Psychiatry, 31: 412-415.

43. Nagayama, T., M. Nagayama, S. Kohara, H. Kamiguchi, M. Shibuya, Y. Katoh, J. Itoh and Y. Shinohara, 2004. Post-ischemic delayed expression of hepatocyte growth factor and c-Met in mouse brain following focal cerebral ischemia. Brain Res., 999: 155-166.

44. Shimamura, M., N. Sato, S. Waguri, Y. Uchiyama, T. Hayashi, H. Iida, T. Nakamura, T. Ogihara, Y. Kaneda and R. Morishita, 2006. Gene transfer of hepatocyte growth factor gene improves learning and memory in the chronic stage of cerebral infarction. Hypertension, 47: 742-751.

45. Badie, B., J. Schartner, J. Klaver and J. Vorpahl, 1999. In vitro modulation of microglia motility by glioma cells is mediated by hepatocyte growth factor/scatter factor. Neurosurgery, 44: 1077-1082; discussion 1082-1083.

46. Zhang, X. and Y. Liu, 2003. Suppression of HGF receptor gene expression by oxidative stress is mediated through the interplay between $\mathrm{Sp} 1$ and Egr-1. Am. J. Physiol. Renal Physiol., 284: F1216F1225.

47. Lavrovsky, Y., B. Chatterjee, R.A. Clark and A.K. Roy, 2000. Role of redox-regulated transcription factors in inflammation, aging and age-related diseases. Exp. Gerontol., 35: 521-532.

48. Bijur, G.N., R.E. Davis and R.S. Jope, 1999. Rapid activation of heat shock factor-1 DNA binding by $\mathrm{H} 2 \mathrm{O} 2$ and modulation by glutathione in human neuroblastoma and Alzheimer's disease cybrid cells. Brain Res. Mol. Brain Res., 71: 69-77.

49. Thiriet, N., J. Zwiller and S.F. Ali, 2001. Induction of the immediate early genes egr-1 and c-fos by methamphetamine in mouse brain. Brain Res., 919: 31-40.

50. Li, Z., S. Mizuno and T. Nakamura, 2007. Antinecrotic and antiapoptotic effects of hepatocyte growth factor on cholestatic hepatitis in a mouse model of bile-obstructive diseases. Am. J. Physiol. Gastrointest. Liver Physiol., 292: G639646.
51. Ekuni, D., J.D. Firth and E.E. Putnins, 2006. Regulation of epithelial cell growth factor receptor protein and gene expression using a rat periodontitis model. J. Periodontal Res., 41: 340349.

52. Kurz, S.M., S.S. Diebold, T. Hieronymus, T.C. Gust, P. Bartunek, M. Sachs, W. Birchmeier and M. Zenke, 2002. The impact of c-met/scatter factor receptor on dendritic cell migration. Eur. J. Immunol., 32: 1832-1838.

53. Masliah, E., N. Ge, M. Morey, R. DeTeresa, R.D. Terry and C.A. Wiley, 1992. Cortical dendritic pathology in human immunodeficiency virus encephalitis. Lab. Invest., 66: 285-291.

54. Brendza, R.P., B.J. Bacskai, J.R. Cirrito, K.A. Simmons, J.M. Skoch, W.E. Klunk, C.A. Mathis, K.R. Bales, S.M. Paul, B.T. Hyman and D.M. Holtzman, 2005. Anti-Abeta antibody treatment promotes the rapid recovery of amyloidassociated neuritic dystrophy in PDAPP transgenic mice. J. Clin. Invest., 115: 428-433.

55. Hasbani, M.J., K.L. Hyrc, B.T. Faddis, C. Romano and M.P. Goldberg, 1998. Distinct roles for sodium, chloride and calcium in excitotoxic dendritic injury and recovery. Exp. Neurol., 154: 241-258.

56. Swann, J.W., S. Al-Noori, M. Jiang and C.L. Lee, 2000. Spine loss and other dendritic abnormalities in epilepsy. Hippocampus, 10: 617-625.

57. Bellizzi, M.J., S.M. Lu, E. Masliah and H.A. Gelbard, 2005. Synaptic activity becomes excitotoxic in neurons exposed to elevated levels of platelet-activating factor. J. Clin. Invest., 115: 3185-3192.

58. Ikegaya, Y., J.A. Kim, M. Baba, T. Iwatsubo, N. Nishiyama and N. Matsuki, 2001. Rapid and reversible changes in dendrite morphology and synaptic efficacy following NMDA receptor activation: implication for a cellular defense against excitotoxicity. J. Cell. Sci., 114: 40834093.

59. McHugh, T.J., K.I. Blum, J.Z. Tsien, S. Tonegawa and M.A. Wilson, 1996. Impaired hippocampal representation of space in CA1-specific NMDAR1 knockout mice. Cell, 87: 1339-1349.

60. Tsien, J.Z., P.T. Huerta and S. Tonegawa, 1996. The essential role of hippocampal CA1 NMDA receptor-dependent synaptic plasticity in spatial memory. Cell, 87: 1327-1338.

61. Raymond, G.V., M.L. Bauman and T.L. Kemper, 1996. Hippocampus in autism: a Golgi analysis. Acta Neuropathol. (Berl), 91: 117-119. 
62. Magee, J.C. and D. Johnston, 1997. A synaptically controlled, associative signal for Hebbian plasticity in hippocampal neurons. Science, 275: 209-213.

63. Markram, H., J. Lubke, M. Frotscher and B. Sakmann, 1997. Regulation of synaptic efficacy by coincidence of postsynaptic APs and EPSPs. Science, 275: 213-215.

64. Dan, Y. and M.M. Poo, 2006. Spike timingdependent plasticity: from synapse to perception. Physiol. Rev., 86: 1033-1048.

65. Bi, G.Q. and M.M. Poo, 1998. Synaptic modifications in cultured hippocampal neurons: dependence on spike timing, synaptic strength and postsynaptic cell type. J. Neurosci., 18: 1046410472.

66. Gibson, J.R., M. Beierlein and B.W. Connors, 1999. Two networks of electrically coupled inhibitory neurons in neocortex. Nature, 402: 7579.

67. Cobb, S.R., E.H. Buhl, K. Halasy, O. Paulsen and P. Somogyi, 1995. Synchronization of neuronal activity in hippocampus by individual GABAergic interneurons. Nature, 378: 75-78.

68. Coull, J.A., S. Beggs, D. Boudreau, D. Boivin, M. Tsuda, K. Inoue, C. Gravel, M.W. Salter and Y. De Koninck, 2005. BDNF from microglia causes the shift in neuronal anion gradient underlying neuropathic pain. Nature, 438: 10171021.

69. Rivera, C., H. Li, J. Thomas-Crusells, H. Lahtinen, T. Viitanen, A. Nanobashvili, Z. Kokaia, M.S. Airaksinen, J. Voipio, K. Kaila and M. Saarma, 2002. BDNF-induced TrkB activation down-regulates the $\mathrm{K}+-\mathrm{Cl}$ - cotransporter $\mathrm{KCC} 2$ and impairs neuronal Cl- extrusion. J. Cell Biol., 159: 747-752.
70. Anderson, M.P., T. Mochizuki, J. Xie, W. Fischler, J.P. Manger, E.M. Talley, T.E. Scammell and S. Tonegawa, 2005. Thalamic Cav3.1 T-type Ca2+ channel plays a crucial role in stabilizing sleep. Proc. Natl. Acad. Sci. USA, 102: 1743-1748.

71. McVicar, K.A. and S. Shinnar, 2004. LandauKleffner syndrome, electrical status epilepticus in slow wave sleep and language regression in children. Ment. Retard. Dev. Disabil. Res. Rev., 10: 144-149.

72. Trevathan, E., 2004. Seizures and epilepsy among children with language regression and autistic spectrum disorders. J. Child Neurol., 19 (Suppl 1): S49-57.

73. Saper, C.B., T.C. Chou and J.K. Elmquist, 2002. The need to feed: homeostatic and hedonic control of eating. Neuron, 36: 199-211.

74. Saper, C.B., T.E. Scammell and J. Lu, 2005. Hypothalamic regulation of sleep and circadian rhythms. Nature, 437: 1257-1263.

75. Hammock, E.A. and L.J. Young, 2006. Oxytocin, vasopressin and pair bonding: implications for autism. Philos. Trans. R. Soc. Lond. B. Biol. Sci., 361: 2187-2198.

76. Billups, B. and I.D. Forsythe, 2002. Presynaptic mitochondrial calcium sequestration influences transmission at mammalian central synapses. J. Neurosci., 22: 5840-5847. 